\title{
Video based Fire Detection using BFO Algorithm with Moving Camera
}

\author{
Noor Safaa \\ Department of Electrical Engineering \\ Assist. Lect. In Al-Mustansyria University \\ Baghdad, Iraq
}

\begin{abstract}
Most of buildings that have the fire detection systems provide limited information from the detectors to fire alarm panels. Therefore, there is a need to improve the quality of the information that is provided for this service. This paper proposed a way to detect the fire immediately by video camera, using a style of Bacterial Foraging Optimization (BFO) technique for controlling the DC motor and image processing. In this paper image processing is used to obtain a suitable data that is introduction to the controller. The registration of all video files that have been captured from the moving camera is then stored in the computer. At the time when a fire is noticed by this system, a fire alarm is given immediately and the location of fire occurrence is shown. Simulink is used in this method for the simulation of fire detection, image processing and the controller.
\end{abstract}

\section{Keywords}

Image processing, BFO, DC motor.

\section{INTRODUCTION}

The fire continually causes economic and environmental damage as well possible the people's lives. So, to avoid these damages many fire-detection techniques have been developed. But, most of these techniques are based on temperature sensors, relative humidity sampling, smoke analysis, ect. [1]. on the other side, those detectors can't provide accurate information about the fire, like its location, size, growing rate, and so on. Therefore, Video Image Detection (VID) is becoming more interesting and considered one of the applications of artificial intelligence to analyze video image that using to detect the fire. VID systems detect the existence of fire within the video images at smaller distances or covering large areas, which provide faster detection with accurate results [1]. Fire detection methods by video processing and the related research work can be found in [26]. In [2], this method represented a fuzzy logic approach to fire detection in aircraft dry bays and engine compartments which are used a set of statistical measures that derived from histograms and image subtraction analysis of the sequence image frames. In $[3,4]$, the method proposed a model for fire color. The authors add their model with moving object in order to detect fires in video; then these objects are determined by the background subtraction technique. [5] Many authors are presented edge-detection methods to set their effectiveness in flame edge identification. For modulation many parameters in the use of these methods, the results were still unacceptable when we apply these methods on color image. The method in [6], proposed a real-time alarm system for detecting flames using the spectral, spatial and temporal features, where these features are weighted with a fuzzy reasoning system to give probable flame candidate regions. This work presented the application of a BFO system to detect fire in early stage based on video image detection.

This paper is organized as follows. The overall fire detecting algorithm structure is introduced and analyzed in section 2. The design of controller is given in section 3. The mathematical model of DC motor describe in section 4. Then some experimental results are given in section 5. Finally, the conclusions and suggestions for future works are given in section 6 .

\section{DETECTION ALGORITHM}

The main program of the system consists of the video input from the camera shown in Figure 4, where the video is stored in PC, 240*320 resolution video 30 frames per second.

Basically, the Simulink of Matlab version 7.8 is used to process the data and image processing, and then use the output data as input to the controller. The process that is included in the image processing steps is the median filter, threshold, image enhancement.

\subsection{Filter Subsystem}

In this part, the enhancement processed is done. Figure 1 shows image is started with the gamma correction block. Gamma correction block is used to apply or remove gamma correction from the input video stream. Typical gamma values range from 1 to 3 [7].

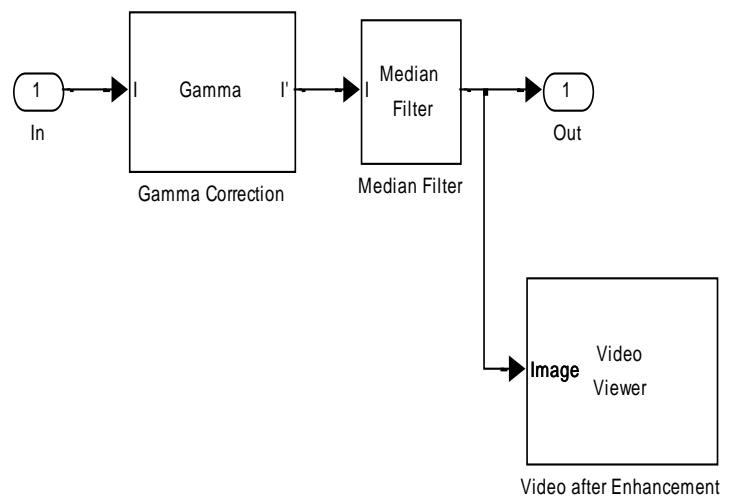

Fig 1: Filter Subsystem 


\subsection{Threshold Subsystem}

They are threshold process, where the image data after enhancement is then converted to white and black colors by using the threshold block. The threshold block is used to convert an intensity image to a binary image using a threshold value [7] which is chosen in this work manually (0.95) after trying several values until reaching the suitable appropriate value. The block computes this manual threshold value by and the variance of each pixel group is minimized.

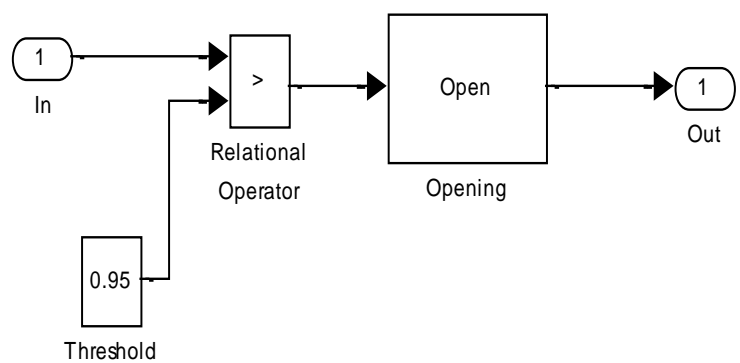

Fig 2: Threshold Subsystem

After the threshold process, the entire image is converted to black and white colors only. From here, the process of the operation will become easier.

\subsection{SAD Computation}

Sum of Absolute Difference (SAD) Computation is used for motion estimation in video image. It is usually the computationally dense part in video processing. Therefore, a method to reduce this complication is carefully required. SAD is an so fast metric because of its simplicity, it is absolutely the simplest possible metric that takes into account every pixel in a block [8]. Therefore it is very efficient for a broad motion search of many various blocks. SAD is facility to analyzes each pixel separately, making the implemented is easy [8]. The motion estimation are needed to reduce the number of bits in the sequential video frames that have similar cases where Most of the sequential video frames will be similar except for the changes that produced by objects moving.

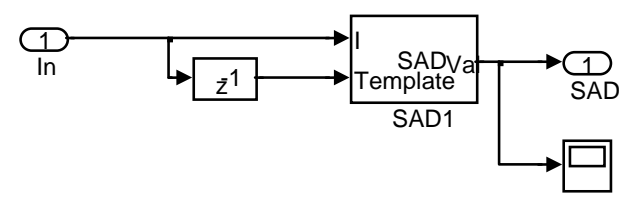

Fig 3: Subsystem of Motion Energy

\section{BFO ALGORITHM}

The Bacterial Foraging Optimization (BFO) by Passino in 2002 is based on foraging theory of E. coli bacteria. The basic
Bacterial Foraging Optimization consists of three major techniques: chemotaxis, reproduction and eliminationdispersal. The brief described of these techniques concerned in Bacterial Foraging are given below [9].

\subsection{CHEMOTAXIS}

A list of random samples will be produced as the initial position of each bacterium, then choosing the bacteria with good foraging impact to increase and removing the bacteria that have bad effect, the individual which has lower fitness value in the solution space is removed [10].

Finally, the bacterial set goes to the new region by a specific probability, which reduces the probability of converging to local optimum and improves the probability to find the optimal solution [11]. Suppose $\theta^{\mathrm{j}}(\mathrm{i}, \mathrm{k}, \ell)$ represents the bacterium at jth chemotactic, kth social, and $\ell$ th removaldispersal step, in each account chemotactic step, the movement of the ith bacterium can be represented as:

$\theta^{i}(i+1, k, \ell)=\theta^{j}(i, k, \ell)+C(j)\left[\Delta(j) /\left(\Delta T j \Delta(j)^{1 / 2}\right]\right.$

Where $\Delta(\mathrm{j})$ is the direction vector of the jth chemotactic step. When the bacterial movement is run, $\Delta(\mathrm{j})$ is the same with the last chemotactic step; otherwise, $\Delta(\mathrm{j})$ is a random vector that each elements lie in $[-1,1]$.

\subsection{Swarming}

When the cells reaching at the better food location it is always required that the bacterium which has searched the optimum path should attempt to supply an signal to other bacteria then they swarm together to reach the wanted location. In this process, the bacteria get together into groups and hence move concentric patterns of groups with high bacterial intensity [12]. These attraction signals are denoted as:

$\operatorname{Jcc}(\theta, \mathrm{P}(\mathrm{j}, \mathrm{k}, \ell))=\sum_{\mathrm{i}=1}{ }^{\mathrm{s}} \mathrm{J}_{\mathrm{cc}}\left(\theta, \theta^{\mathrm{i}}(\mathrm{j}, \mathrm{k}, \ell)\right)=\sum_{\mathrm{i}=1} \mathrm{~s}\left[-d_{\text {attract }}\right.$ $\exp \left[-\right.$ Wattract $\left.\left.\sum_{\mathrm{m}=1} \mathrm{p}\left(\theta_{\mathrm{m}}-\theta_{\mathrm{m}}^{\mathrm{i}}\right)^{2}\right)\right]+\sum_{\mathrm{i}=1}{ }^{\mathrm{s}}\left[-h_{\text {repellant }} \exp \right.$ $\left(-\right.$ Wrepellant $\left.\left.\sum_{\mathrm{m}=1}^{\mathrm{p}}\left(\theta_{\mathrm{m}}-\theta_{\mathrm{m}} \mathrm{i}\right)^{2}\right)\right]$

Where $\operatorname{Jcc}(\theta, P(j, k, \ell))$ is the objective function value to be added to the current objective function to siting time changing objective function, $\mathrm{S}$ is the total number of bacteria, $\mathrm{P}$ is the number of variables interested in the search space, $\theta=[\theta 1, \theta 2$, $\ldots, \theta \mathrm{P}] \mathrm{T}$ is a point on the optimization domain, and $\theta \mathrm{mi}$ is the mth components of the ith bacterium position $\theta \mathrm{i}$. [12].

\subsection{Reproduction}

The bacteria that have lowered healthy will die and each healthier bacterium is divided into two bacteria, then each one located in the same position [12].

\subsection{Elimination and Dispersal}

The elimination and dispersion represents the appearance of sudden incidents which can bring changes in the evolution process. This type of unexpected occurrence is mostly occurrence of unknown events that may exchange a list of bacteria to a location closer to the food location [12] 


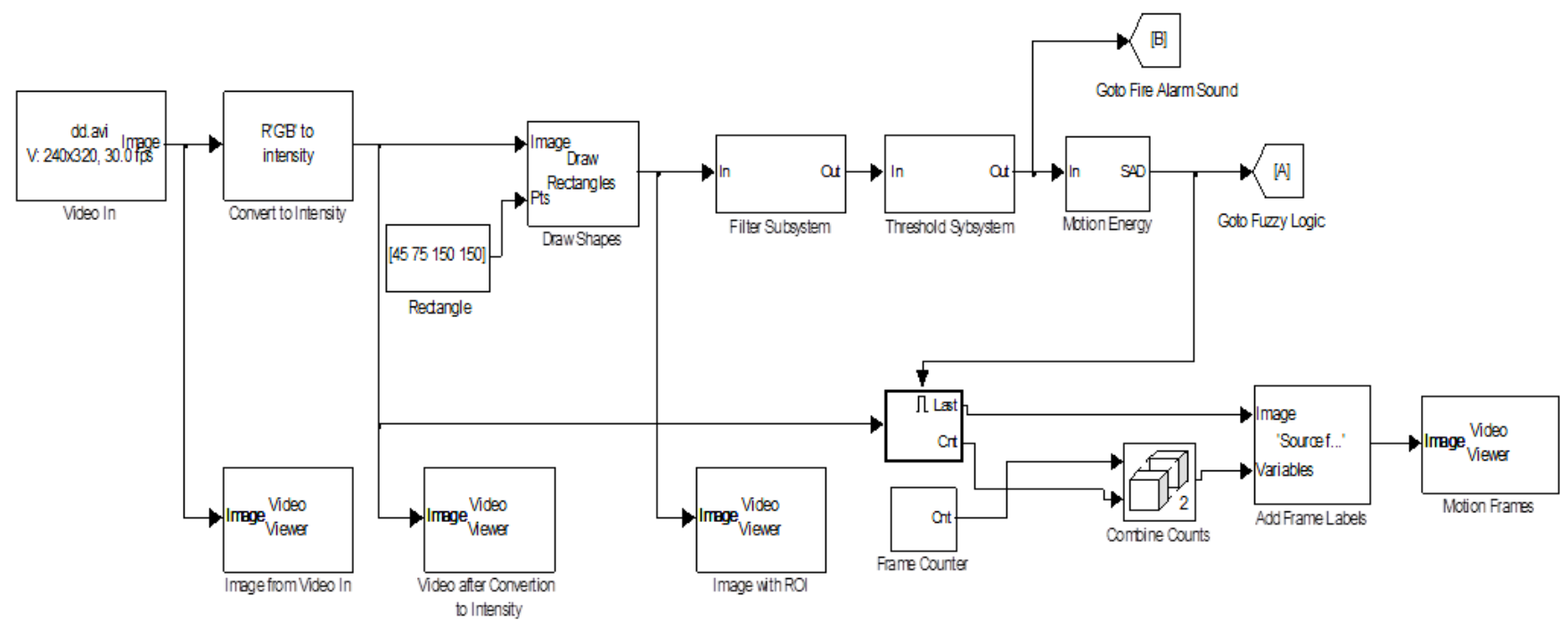

Fig 4: The main program of image processing

\section{THE DC MOTOR}

The DC motor subsystem is the main component that decides the position of object [13]. This subsystem is connected mechanically to the camera movement of the designed system. The main idea in this simulation is to observe how the position of the system can be controlled and how does a controller affect the system. In this work, DC motor model was chosen for the control system design. The DC motor subsystem model is designed using Simulink block diagram as shown in the Figure 5. A few blocks are set as constant parameters namely the $\mathrm{K}$ (constant), $\mathrm{R}$ (electric resistance), $\mathrm{J}$ (moment of inertia of the rotor), $\mathrm{L}$ (electric inductance) and $\mathrm{b}$ (damping ratio of the mechanical system) where:
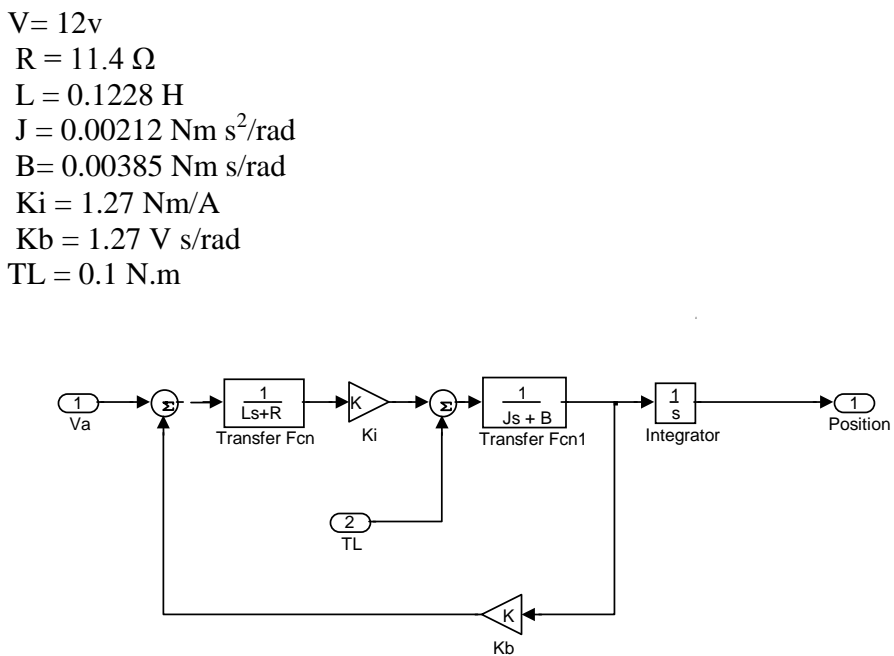

Fig 5: Simulink model of DC motor

\section{THE SIMULATION RESULTS}

The simulation is doing using MATLAB tool box. This work is based on BFO technique for position control of DC motor. DC motor is loading with different loads to see the performance of the designing controller.
By applying the algorithm of image processing with the controller on the video candle with lighting a lamp, where the results are shown below.

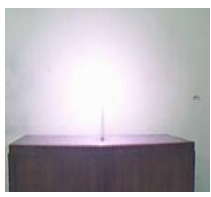

Video in

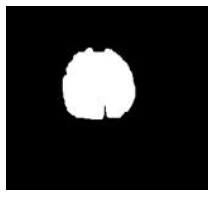

Video after Th

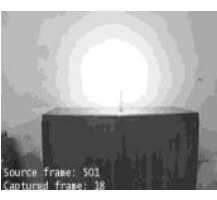

Video with motion
Fig 6: Video of a candle burning with lighting lamp

The result of SAD is shown in Figure 7, where the resultant of $\mathrm{SAD}$ value is zero when there are no changes in the video frames motion which leads to a value of zero, and one when the video frames are changed due to the lighting of the candle which induces by fire motion.

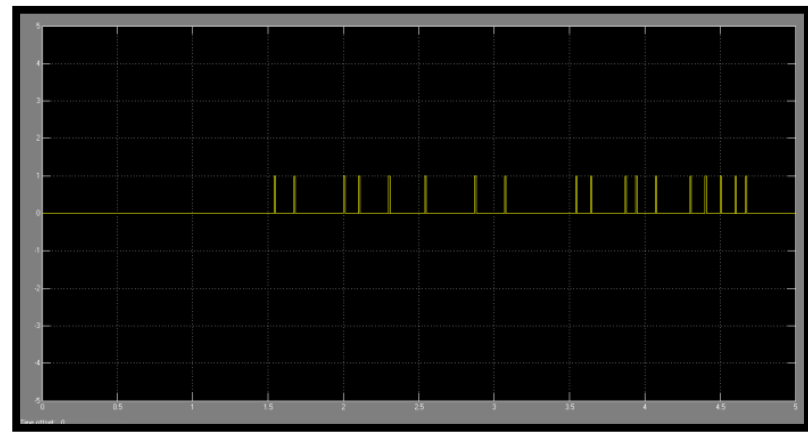

Fig 7: The output of SAD video of candle with a lighting lamp 


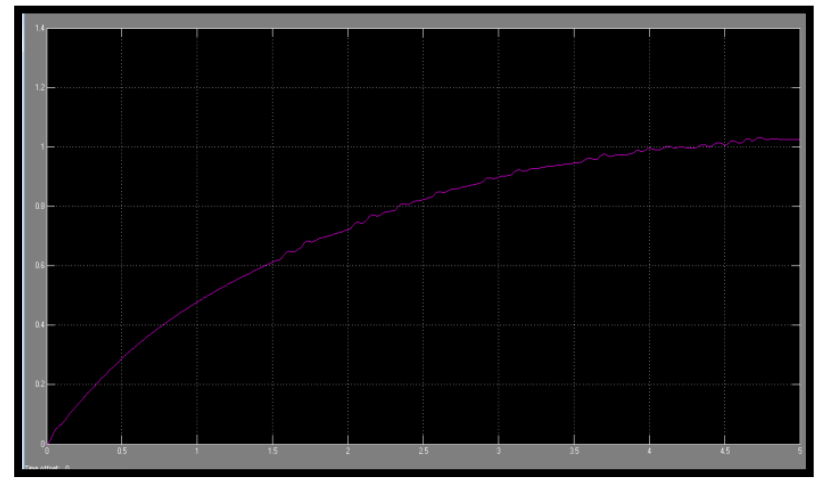

Fig 8: The output position in case of candle with a lighting lamp

The output position is represented in Figure 8, where the DC motor continues to rotate when the background is black, i.e., when there is no motion of fire and stops when the fire occurred. In this case, position is estimated to be the angle where fire is occurred as shown in Figure 8. A desired for the controller is $1.029 \mathrm{rad}$ equal to 59.014 degree after $4.8 \mathrm{sec}$, another case of video without flame candle.

By taking another case of video candle burning in dark, from Figure 9, it is seen that, the video after threshold occurs white pixels, this denotes that there is a fire, but smaller than the white pixels which occur with the video candle with light. In fact that shows the effect of luminance on the detection. And, the result of SAD shown in Figure 10, where the video frames are similar, and then the resultant is still zero and becomes one value when the fire occurs. Also, the effect of luminance shows on the result of SAD compared with Figure 10 about the video frames.

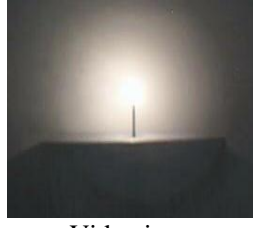

Video in

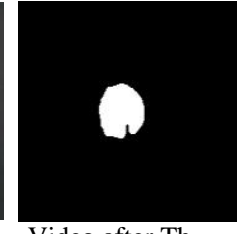

Video after Th

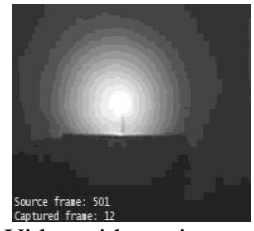

Video with motion
Fig 9: Video candle burning in dark

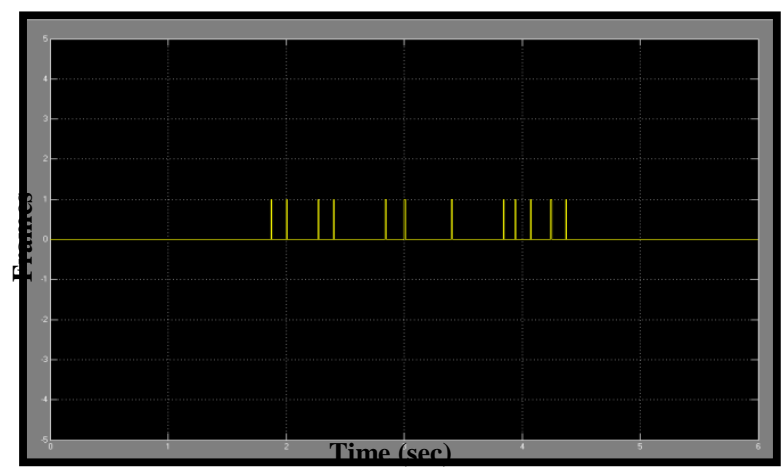

Fig 10: The output of SAD video of candle burning in dark

Also, the DC motor continues to rotate when the background is black, i.e., when there is no motion of fire and stops when the fire occurs, but with shorter time at $4.4 \mathrm{sec}$ than the video of candle with light.

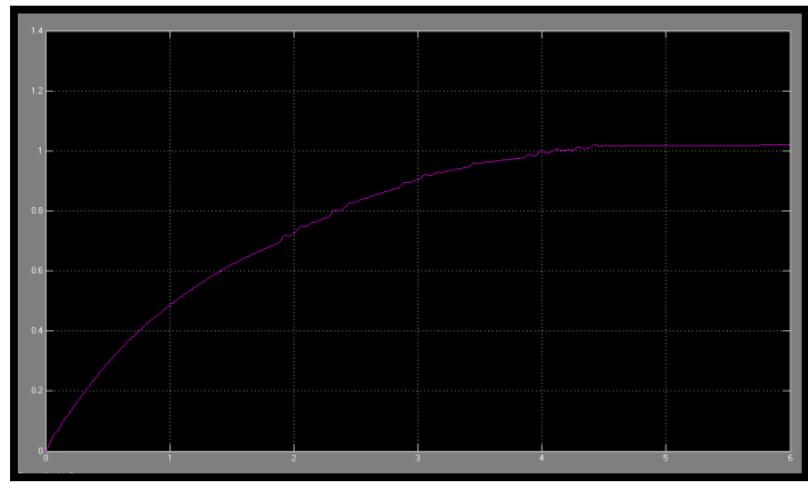

Fig 11: The output position of candle burning in dark

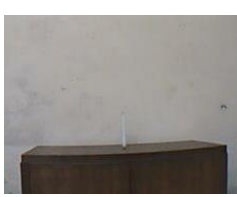

Video in

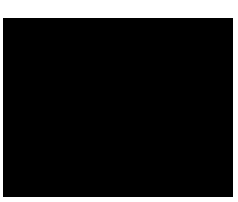

Video after Th

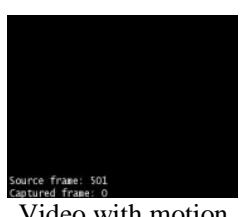

Video with motion
Fig 12: Video of candle without burning

The video (when there is no fire) in Figure 12 shows only dark when there is no fire. And the frame motion still dark as there is no motion. Figure 13 shown the resultant of SAD is zero when the video frames is similar, which denote that there is no fire, this result will be send to the controller to show the position of fire in this case, where Figure 14 shown the output position when the DC motor continue rotating without stopped when there is no fire.

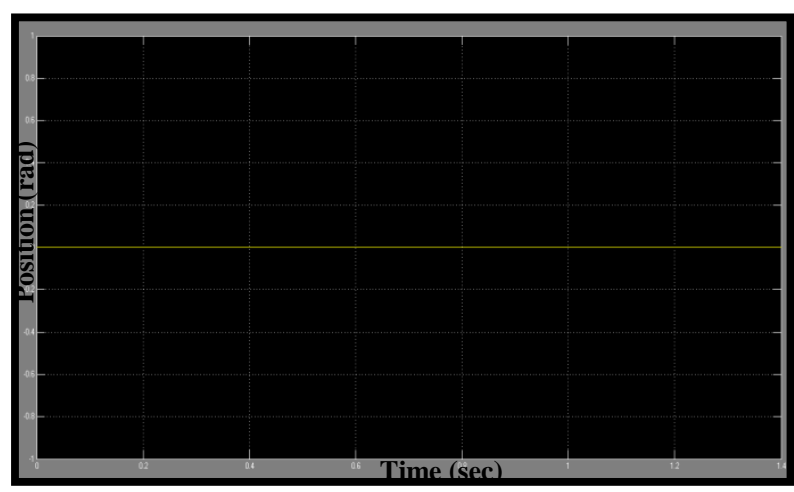

Fig 13: The output of SAD video candle without burning

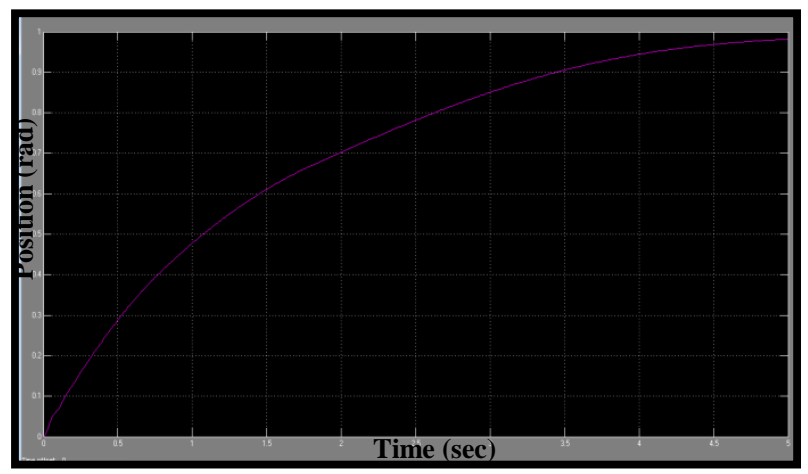

Fig 14: The output position of candle in dark case where the DC motor continues rotating 
In the case of fire occurrence, a suitable voice of warning will be heard. Figure 15 shows the warning process indicating that there is a fire.

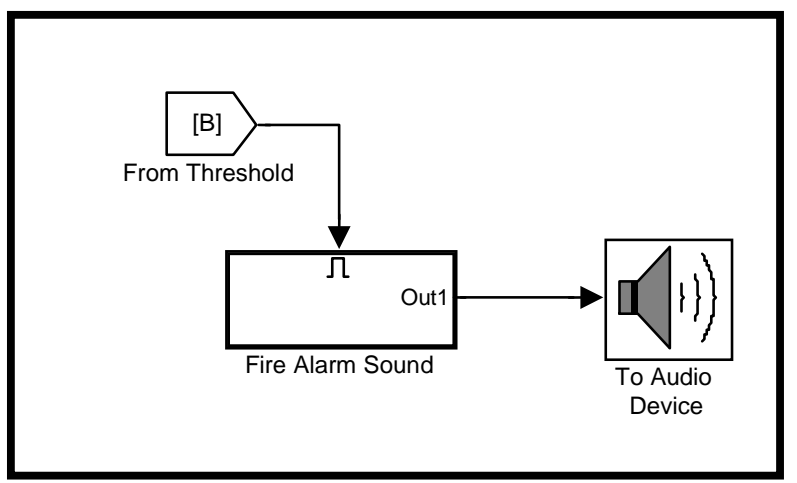

Fig 15: Fire alarm sound

\section{CONCLUSIONS AND FUTURE WORK}

The general conclusions and simulation results are based on the simulation carried out with the aid of Matlab software version 7.8. In fire detection method, the BFO technique was designed and implemented to control a DC-motor, to maintain the camera facing the fire. The evaluated fire VID systems, equipped with multiple image sources, intelligent image recognition and processing algorithm, were able to detect various cases of fires at their early stages. They were impenetrable from various disturbance sources, such as artificial lights. The simulation results show that fire can be successfully detected under various environmental conditions, i.e., indoor, with and without lighting, simple or complex background image, etc. Video processing, uses the SAD (Sum of Absolute Differences) for motion estimation in video, it is effective simplest possible method which takes into account every pixel in a block. As a suggestion for future work, the use of neural-fuzzy method will extend the monitoring and control of fire safety systems outside of buildings can be monitored at any time and from anywhere.

\section{REFERENCES}

[1] Thou-Ho Chen and Chao-Yu Chen, "An Intelligent RealTime Fire-Detection for DVR System", Department of Electronic Engineering, China, 2005.

[2] S.Y. Foo, "A fuzzy logic approach to fire detection in aircraft dry bays and engine compartments", IEEE Trans. Industrial Electronics, vol. 47, no. 5, pp. 1161-1171, 2000 .
[3] Celik, T., Demirel, H., Ozkaramanli, H., Uyguroglu and M., "Fire Detection in Video Sequences Using Statistical Color Model", Proc. International Conference on Acoustics, Speech and Signal Processing, Vol. 2, pp. 213-216, May 2006.

[4] Celik, T., Demirel, H., Ozkaramanli and H., "Automatic Fire Detection in Video Sequences", IEEE International Conference, Dept. of Electr. \& Electron. Eng., Eastern Mediterranean Univ., Turkey, Sep. 2006.

[5] Mr. Sahil Raheja, " An Edge Detection Approach for Flame and Fire Images in Image Processing Using Bacterial Foraging Algorithm ", (IJERT), ISSN: 22780181, Vol. 3 Issue 7, July - 2014.

[6] C-C-Ho, "Machine vision-based real time early flame and smoke detection", IEEE International Conference, Meas. Sci. Tech., China, Vol. 20, pp. 1-13, 2009.

[7] Mathworks, 2009.

[8] Riza Muhida Eklas Hossain, Ahmed Faris and Ahad Ali, "A Vision-Based Sun Tracking System to Increase Performance of Solar Cells", International Conference on Instrumentation, Control and Automation, Indonesia, Oct. 2009.

[9] Chen Li, "Application of Bacterial Foraging Optimization PID Control in VAV System", Shandong Normal University School of Information Science \& Engineeringm JiNan China, ICCSEE, 2013.

[10] Turgay Celik, Huseyin Ozkaramanli and Hasan Demirel, "Fire Pixel Classification Using Fuzzy Logic and Statistical Color Model", Department of Electrical and Electronic Engineering, Eastern Mediterranean University, Turkey, 2007.

[11] Fan Feizhi. "the Research and improve of BFO algorithm". North China Electric Power University, master's degree thesis. 2010.

[12] Ahmed H. Abo absa and Mohammed A. Alhanjouri, "PID PARAMETERS OPTIMIZATION USING BACTERIA FORAGING ALGORITHM AND PARTICLE SWARM OPTIMIZATION TECHNIQUES FOR ELECTROHYDRAULIC SERVO CONTROL SYSTEM", The 4th International Engineering Conference Towards engineering of $21^{\text {st }}$ century, 2012.

[13] Dr. Ismail H. Altas, "DC Motor Position Control", Electrical and Electronics Engineering, Karadeniz Technical University, Turkey, 2007. 